\title{
Orientation Imaging Microscopy of Nanocrystalline Platinum Thin Film Using Transmission Electron Microscope
}

\author{
V. Kumar* \\ * Department of Metallurgical Engineering, University of Utah, Salt Lake City, UT-84102
}

Orientation imaging microscopy (OIM) provides crystallographic orientations of crystals in the microstructure on a regular grid of points. The crystallographic orientations can be used to obtain, grain size, texture, and grain boundary characteristics of the material sample. Orientation imaging microscopy (OIM) is usually performed using electron back scattered diffraction (EBSD) technique in scanning electron microscope (SEM). The spatial resolution of EBSD technique is not adequate to provide high quality OIM data for nanocrystalline material samples with sub-100 $\mathrm{nm}$ grain size [1]. Therefore, transmission electron microscope (TEM) is used for performing orientation imaging microscopy (OIM) on nanocrystalline materials. Orientation imaging microscopy (OIM) in TEM can be performed using one of the three available methods: (1) conical scan method, (2) microdiffraction method and (3) Kikuchi method. While these methods can provide OIM data, authors in [1-3] argued that these methods do not perform satisfactorily. In this paper, an automated method to perform orientation imaging microscopy (OIM) in TEM is presented. The method of this paper utilizes wide angle convergent beam electron diffraction (WACBED) patterns. The presented WACBED method is applied to nanocrystaline platinum thin film.

For performing orientation imaging microscopy (OIM) using WACBED method, the convergence angle was first optimized to record electron diffraction patterns. The optimization was performed by simulating diffraction patterns using dynamical theory of diffraction. The simulation was performed for randomly oriented 50 grains at increasing convergence angle. Fig. 1 shows the percentage of suitable diffraction patterns as a function of the convergence angle. A suitable diffraction pattern for OIM contains diffraction disks from of at least two families of crystal planes without any disk overlap. For this paper, a convergence angle of 0.55 milliradians was chosen. With optimized convergence angle, diffraction patterns were recorded on a sputtered deposited and annealed $35 \mathrm{~nm}$ thick platinum thin film sample. The diffraction patterns were recorded on a 50 X 50 regular grid of points with $9 \mathrm{~nm}$ spacing using TECHNAI F30 TEM at $300 \mathrm{kV}$ accelerating voltage and 12 spot size.

The positions of diffraction disks in the diffraction patterns were extracted using several image processing steps. The image processing steps include thresholding routines where pixels with intensity greater than a threshold were set to value 1 and rest of the pixels were set to 0 . The diffraction disks were defined as contiguous regions of nonzero value pixels. The geometric centers of the high intensity diffraction disks were used for indexing the diffraction patterns to obtain the orientation information.

Indexing of a diffraction pattern refers to correctly assigning diffraction disks with corresponding diffracting crystal planes. The correct assignment of crystal planes refers to the correct solution of the diffraction pattern. For indexing a diffraction pattern, all possible crystal planes are assigned to diffraction disks. This assignment resulted in a large set of solutions. The solutions in the set were tested by comparing the length of reciprocal lattice vectors (distance between central disk and 
diffracted disks) in the diffraction pattern with the inter-planer spacing of assigned crystal planes. The diffraction patterns were then tested by comparing the angle between the reciprocal lattice vectors with angle between assigned crystal planes. The diffraction patterns qualifying the above two test conditions were considered as the correct solution. If there were more than one correct solutions, a comparison between the simulated diffraction patterns at all possible crystal orientations and correct solutions was performed. The solution with best match in the comparison was taken as the correct solution. Three Euler angles describing the crystallographic orientation of the crystal were calculated from the solution of diffraction pattern. The same procedure is performed on all of the diffraction patterns to obtain orientation information on a regular grid of points in the microstructure. The orientation information can be represented in two dimensions as an orientation map.

Fig. 2(a) shows the $\left[\begin{array}{lll}0 & 0 & 1\end{array}\right]$ inverse pole figure orientation map obtained from WACBED method described above. Fig. 2(b) shows the bright field micrograph with OIM region outlined by a square. In a qualitative visual inspection, the same grain structure can be observed in the orientation map and bright field micrograph. Most of the grains in the OIM map are blue in color which corresponds to [1 1 1] texture. The observed texture using presented WACBED method is identical to the texture of platinum thin film reported in the literature [1]. The comparison of the results with the literature shows that orientation imaging microscopy can be performed satisfactorily on nanocrystalline materials with sub-100 $\mathrm{nm}$ grain size using presented WACBED method.

References

[1] A. Darbal, K Barmak, D. J. Dingley, G. Meaden, J. Michael, T. Sun, B. Yao, K. R. Coffey, Microsc Microanal 15 (suppl 2) (2009) 1232.

[2] S. Zaefferer, G. Wu, Proc. of Int. Conf. on Tex. of Mater. - 15 (2008) 221.

[3] S. Zaefferer, G. Wu, ultramicroscopy, 109 (2009) 1317.

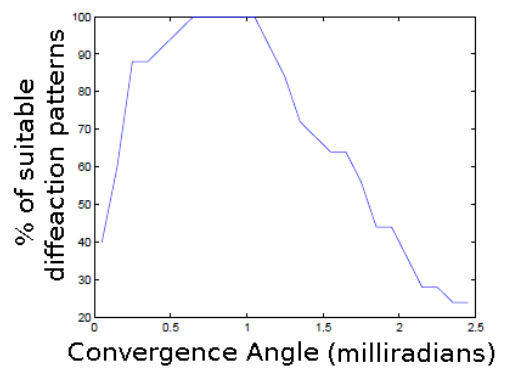

Fig. 1. optimization of convergence angle

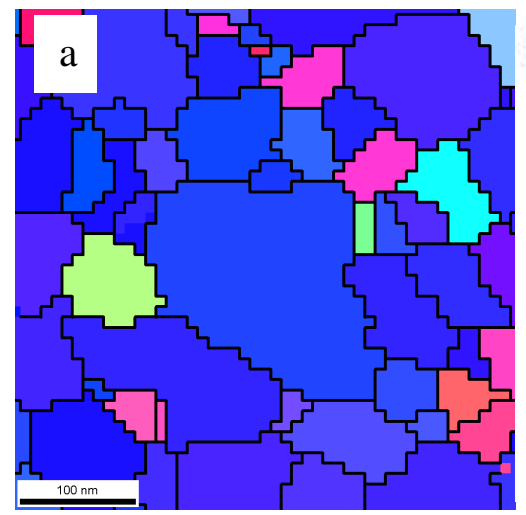

Inverse Pole Figure [001]

Platinum
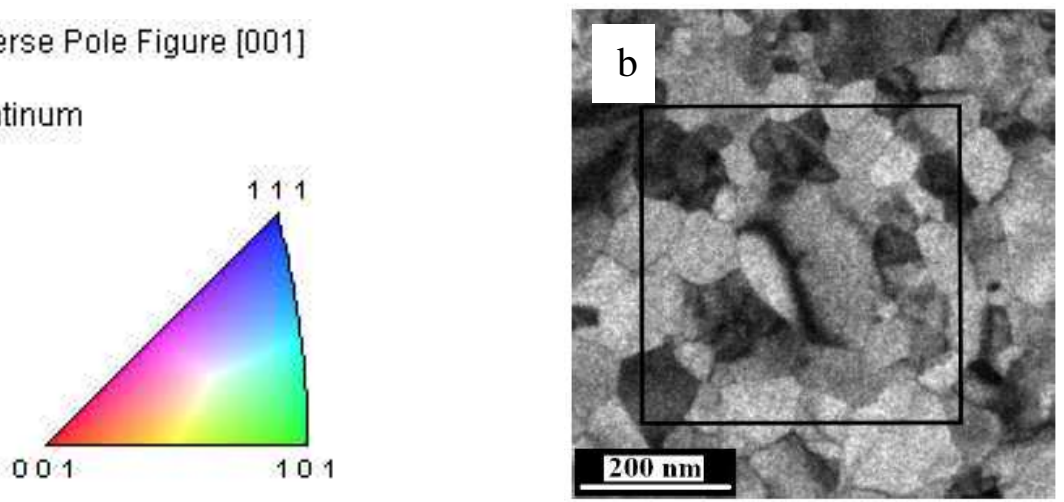

Fig. 1: (a) [ $\left[\begin{array}{lll}0 & 0 & 1\end{array}\right]$ inverse pole figure orientation map, (b) electron micrograph of OIM region 